\title{
THE ASYMPTOTIC BEHAVIOR OF PROPERLY EMBEDDED MINIMAL SURFACES OF FINITE TOPOLOGY
}

\author{
DAVID HOFFMAN AND WILLIAM H. MEEKS III
}

\section{INTRODUCTION}

While advances have been made in recent years in the study of properly embedded minimal surfaces of finite topology in $\mathbf{R}^{3}[3,5,8,9,11,12,13,23]$, progress has depended, in an essential manner, on the special structure of such surfaces with finite total curvature. A properly immersed minimal surface with finite total curvature is, conformally, a compact Riemann surface punctured in a finite number of points, and its Gauss map extends to the compact surface as a meromorphic function [22]. In particular, all the topological ends are conformally equivalent to a punctured disk, and there is a well-defined limit tangent plane at each end. Outside of a sufficiently large compact set, such an end is a multisheeted graph over the limit tangent plane, and if the end is embedded, it is asymptotic to either a plane or a half-catenoid. (See, e.g., Schoen [24]. For a survey of results on properly immersed surfaces of finite total curvature, see $[9, \S 1]$.)

Without the assumption of finite total curvature, these results are not true in general. As an indication of the relative lack of information, consider that it is unknown whether or not the helicoid is the only simply-connected minimal surface that is properly embedded and nonplanar (a question raised by Osserman). The helicoid has infinite total curvature and a single annular end. ${ }^{1}$

If a surface has finite topology, then all of its ends are annular. Any general theory of properly embedded minimal surfaces of finite topology must include an understanding of the behavior of these ends. In this paper, we prove

The Annular End Theorem (Theorem 1 in $\S 4)$. On a properly embedded minimal surface in $\mathbf{R}^{3}$, at most two distinct annular ends can have infinite total curvature.

All other annular ends have finite total curvature and are therefore geometrically well behaved (i.e., asymptotic to the plane or a half-catenoid).

As a simple consequence of this result, a properly embedded minimal surface of finite topology in $\mathbf{R}^{3}$ must be conformally equivalent to a closed Riemann

Received by the editors August 19, 1988.

1980 Mathematics Subject Classification (1985 Revision). Primary 53A10.

The research in this paper was supported by research grant DEFG02-86ER250125 of the Applied Mathematical Science subprogram of the Office of Energy Research, U. S. Department of Energy, and National Science Foundation grants DMS-8802858 and DMS-8611574.

${ }^{1}$ By annular, we mean homeomorphic to a punctured disk. 
surface from which a finite number of points and zero, one, or two compact disks have been removed (Corollary 4 in $\S 4$ ).

We conjecture that on properly embedded minimal surfaces in $\mathbf{R}^{3}$ with more than one end, all annular ends have finite total curvature. If this conjecture is true, it would follow that a properly embedded minimal surface with more than one end has finite total curvature if and only if it has finite topology. ${ }^{2}$

The paper is organized as follows. In $\S 2$, special foliations of solid tori by compact minimal annuli are constructed (Proposition 1). These are necessary for use in $\S 3$ where we show that, under proper conditions, linear bounds on the growth of an annular end imply that the end has finite total curvature (Lemma 4 of $\S 3)$. This result, which we refer to as "the Cone Lemma," is the key component in the proof of the Annular End Theorem in $\S 4$. Some of these results were announced in [13].

Other related results. By using techniques developed in [17], it is possible to generalize the proof of the Annular End Theorem to show that on a properly embedded minimal surface $M$ with more than one end, any annular end, $E$, of infinite total curvature has boundary curve $\partial E$ that is homotopically trivial in the closure of exactly one of the components of $\mathbf{R}^{3}-M$.

In [4], it is proved that every annular end of a complete orientable nonsimplyconnected minimal surface has a unique representative, called a canonical end, whose boundary is a closed geodesic. Distinct canonical ends have disjoint interiors. Moreover, if their boundaries touch, they must coincide. This means that, except when $M$ is an annulus, canonical ends have disjoint closures. Using this result and the Annular End Theorem, the authors and M. Callahan have proved that a properly embedded minimal surface with more than one end, whose symmetry group is infinite, is either the catenoid or has an infinite number of ends, and all the annular ends are flat [4].

Fang and Meeks [6] have used the Annular End Theorem to prove that a properly embedded minimal surface $M$ with a catenoid-type end can have, at most, one end of infinite total curvature.

In the study of periodic, embedded minimal surfaces, one considers complete minimal surfaces in $\mathbf{R}^{3} / T$, where $T$ is a discrete group of isometries. In this context, Meeks and Rosenberg have shown that a properly embedded minimal surface in a complete nonsimply-connected flat three-manifold has finite topology if and only if it has finite total curvature [16, 18].

Acknowledgments. We wish to acknowledge our debt to R. Osserman who initiated the study of global properties of complete minimal surfaces of finite total curvature. As we grope with the difficulties of infinite total curvature, it is increasingly clear to us how much we have been fortunate enough to take for

\footnotetext{
${ }^{2}$ This would, in turn, imply that a properly embedded minimal surface of finite topology and infinite total curvature would have to have one end. One might then be tempted to conjecture that the helicoid was the only properly embedded minimal surface of finite topology and infinite total curvature. Of course, it is possible to conjecture just about anything in the absence of examples; before the papers referred to in the first paragraph, in was generally believed that the only properly embedded minimal surfaces of finite total curvature were the plane and the catenoid. Still, if there really are no other examples...
} 
granted. The authors also wish to thank Yi Fang for useful critical comments and James Hoffman for preparing the illustrations.

\section{SPECIAL FOLIATIONS OF SOLID TORI}

Let $\alpha$ be a smooth Jordan curve contained in a plane $P$. A disk in $P-\alpha$ is maximal if it is not contained in any larger disk in $P-\alpha$. We define $r_{\alpha}$ to be the infimum of the radii of all maximal disks. Clearly, $r_{\alpha}$ is positive. Moreover, an $\varepsilon$-neighborhood of $\alpha$ is a regular neighborhood if $\varepsilon<r_{\alpha}$.

Remark 1. If $\alpha_{t}$ is a smooth deformation of $\alpha, r_{\alpha_{t}}$ is continuous in $t$.

Now assume that $\alpha$ lies in the $\left(x_{1}, x_{2}\right)$-plane in $\mathbf{R}^{3}$. Again, the tubular neighborhood, $T^{\varepsilon}$, of $\alpha$ is a regular neighborhood if $\varepsilon<r_{\alpha}$. A simple calculation shows that $\partial T^{\varepsilon}$ will have positive mean curvature if $\varepsilon<r_{\alpha} / 2$. In what follows, we will always assume that $\varepsilon<r_{\alpha} / 2$. For convenience, we will refer to the plane $\left\{x_{3}=\varepsilon\right\}$ as $P^{\varepsilon}$. Let $\alpha^{\varepsilon} \subset P^{\varepsilon}$ be the vertical translate by $\varepsilon$ of $\alpha \subset P^{0}$. Note that $\alpha^{-\varepsilon} \cup \alpha^{\varepsilon} \subset \partial T^{\varepsilon}$.

Let $\Sigma \subset T^{\varepsilon}$ be any compact minimal surface with $\partial \Sigma=\alpha^{-\varepsilon} \cup \alpha^{\varepsilon}$.

Lemma 1. Given $\eta>0$, there exists an $\varepsilon_{1}>0$ such that if $\varepsilon<\varepsilon_{1}$, the geodesic curvature of $\partial \Sigma=\alpha^{-\varepsilon} \cup \alpha^{\varepsilon}$ is bounded by $\eta$ in absolute value. Moreover, $\varepsilon_{1}=\varepsilon_{1}(\eta)$ is independent of $\Sigma$ and depends only on $r_{\alpha}$ (and, of course, $\eta$ ).

Proof. We note first that the curvature of $\alpha$ is everywhere bounded by $1 / r_{\alpha}$. The curvature vector of $\alpha^{ \pm \varepsilon}$ lies in $P^{ \pm \varepsilon}$, a horizontal plane. Therefore, the lemma will follow provided we can show that the tangent planes to $\Sigma$ along $\alpha^{\varepsilon} \cup \alpha^{-\varepsilon}$ are uniformly close to vertical for $\varepsilon$ sufficiently small.

Choose $\varepsilon_{1}<r_{\alpha} / 4$. Make $\varepsilon_{1}$ smaller, if necessary, in order to insure the following. Two parallel circles of radius $t \geq r_{\alpha} / 4$ that are separated by a distance $d<2 \varepsilon_{1}$ span a stable catenoid. Choose $\varepsilon<\varepsilon_{1}$.

Fix $p \in \alpha$. There exist two circles in $P^{0}$ that are tangent to $\alpha$ at $p$, have radius $r_{\alpha} / 2$, and meet $\alpha$ only at $p$. Let $c_{1}$ and $c_{2}$ be the centers of these circles, and let $S_{1}(t)$ (resp. $S_{2}(t)$ ) be the circle in $P^{0}$ centered at $c_{1}$ (resp. $c_{2}$ ) of radius $t, r_{\alpha} / 4 \leq t \leq r_{\alpha} / 2$. Let $S_{1}^{ \pm \varepsilon}(t) \subset P^{ \pm \varepsilon}$ be the vertical translate of the circle $S_{1}(t)$. (Similarly, define $S_{2}^{ \pm \varepsilon}(t)$.) By our choice of $\varepsilon_{1}, S_{1}^{\varepsilon}(t) \cup S_{1}^{-\varepsilon}(t)$ bounds a stable catenoid, $C_{1}(t), t \geq r_{\alpha} / 4$. By the convex hull property, the catenoid $C_{1}\left(r_{\alpha} / 2\right)$ is the only catenoid in the family $C_{1}(t)$ whose boundary meets $\alpha^{\varepsilon} \cup \alpha^{-\varepsilon} \subset \partial T^{\varepsilon}$. Also, $C_{1}\left(r_{\alpha} / 4\right)$ is disjoint from $\Sigma$, since it is disjoint from $T^{\varepsilon}$. Hence, by the maximum principle, $C_{1}\left(r_{\alpha} / 2\right)$ meets $\Sigma$ only at $p^{ \pm \varepsilon}$, the vertical translates of $p$.

The same construction works, of course, for the parallel circles $S_{2}^{\varepsilon}(t)$ and $S_{2}^{-\varepsilon}(t)$. The tangent planes to $\Sigma$ at $p^{ \pm \varepsilon}$ are sandwiched between those of $C_{1}\left(r_{\alpha} / 2\right)$ and $C_{2}\left(r_{\alpha} / 2\right)$ at $p^{ \pm \varepsilon}$. By making $\varepsilon_{1}$ suitably small, we can force the tangent planes of the catenoids to be arbitrarily close to vertical. Since the size of such an $\varepsilon_{1}$ is independent of $p$, we are done. 
Lemma 2. There exists an $\varepsilon_{2}>0$ such that for every $\varepsilon<\varepsilon_{2}, \alpha^{\varepsilon} \cup \alpha^{-\varepsilon}$ is the boundary of a minimal annulus in $T^{\varepsilon}$ and every such annulus is stable. The constant $\varepsilon_{2}$ depends only on $r_{\alpha}$ and $L_{\alpha}$, the length of $\alpha$.

Proof. We choose $\varepsilon_{2}=\varepsilon_{1}\left(\pi /\left(2 \cdot L_{\alpha}\right)\right)$, where $\varepsilon_{1}$ is given in the previous lemma. In particular, $\varepsilon_{2}<r_{\alpha} / 2$, so $\partial T^{\varepsilon}$ has positive mean curvature if $\varepsilon<\varepsilon_{2}$. Also, $\alpha^{\varepsilon} \cup \alpha^{-\varepsilon} \subset \partial T^{\varepsilon}$, and $\alpha^{\varepsilon}$ is homotopic to $\alpha^{-\varepsilon}$ in $T^{\varepsilon}$. A geometric version of Dehn's lemma [21] states the following. In a compact Riemannian threemanifold $N$ whose boundary $\partial M$ has nonnegative mean curvature, two disjoint, simple closed curves in $\partial N$, which are homotopic in $N$, must bound a least-area embedded minimal annulus. Thus, $\alpha^{\varepsilon} \cup \alpha^{-\varepsilon}$ bounds a least-area minimal annulus $A \subset T^{\varepsilon}$.

We now prove that any compact minimal annulus $\Sigma \subset T^{\varepsilon}$ with $\partial \Sigma=\alpha^{\varepsilon} \cup \alpha^{-\varepsilon}$ must be stable. By choice of $\varepsilon_{2}=\varepsilon_{1}\left(\pi /\left(2 \cdot L_{\alpha}\right)\right)$,

$$
\int_{\partial \Sigma}\left|\kappa_{g}\right| d s<\frac{\pi}{2 \cdot L_{\alpha}} \int_{\partial \Sigma} d s=\pi
$$

Since $\Sigma$ is an annulus, we may use this inequality, together with the GaussBonnet formula to show that

$$
\int_{\Sigma} K d A=\int_{\partial \Sigma} \kappa_{g} \geq-\pi
$$

from which it follows that $\Sigma$ must be (strictly) stable [2].

We shall prove in Lemma 3 that the stability property of minimal annuli given in Lemma 2 actually implies that there is a unique minimal annulus $A$ in $T^{\varepsilon}$ whose boundary is $\alpha^{\varepsilon} \cup \alpha^{-\varepsilon}$. We note that this uniqueness property for $A$ will not be used in our future constructions in this paper.

Lemma 3. The minimal annulus given in Lemma 2 is unique.

Proof. Let $M$ be the space of smooth, incompressible, embedded minimal annuli in $T^{\varepsilon}$ with boundary curves on $\partial T^{\varepsilon}$. Let $\Pi: M \rightarrow C$ be the projection of $M$ onto the space of pairs of disjoint, embedded, simple closed curves in $\partial T^{\varepsilon}$. By Theorem 2 in [21], an annulus $\Sigma \in M$ meets $\partial T^{\varepsilon}$ transversely along $\partial \Sigma=\Pi(\Sigma) \in C$. Using this fact, it can be shown that $M$ is a smooth manifold. (See [27] for details in a similar case.) Moreover, strict stability of any $\Sigma \in M$ with $\partial \Sigma=\alpha^{-\varepsilon} \cup \alpha^{\varepsilon}$ implies that $\alpha^{-\varepsilon} \cup \alpha^{\varepsilon}$ is a regular value of $\Pi$.

The pair of curves $P^{0} \cap \partial T^{\varepsilon}$ in $C$ bounds the flat domain $P^{0} \cap T^{\varepsilon}$, which is the unique minimal annulus with this boundary. Therefore, this pair is also a regular value of $\Pi$. Let $\Gamma:[0,1] \rightarrow C$ be an embedded, smooth path in $C$, beginning at $\Gamma(0)=\alpha^{-\varepsilon} \cup \alpha^{\varepsilon}$ and ending at $P^{0} \cap T^{\varepsilon}$. Since $\Pi$ is a Fredholm map of index zero, the Smale transversality theorem [26] allows us to choose $\Gamma$ transverse to $\Pi$. Since the annuli in $M$ are incompressible, the length of any curve representing a generator of $\pi_{1}(A), A \in M$, must be greater than a positive constant that depends only on $\varepsilon$. This property easily allows one to generalize the proof of the compactness theorem in [1] to show that $\Pi$ is proper. Therefore, $\Pi^{-1}(\Gamma)$ consists of a finite number of embedded closed 
curves in $M$ and a finite number of compact arcs. Any endpoint of these arcs must be either $P^{0} \cap T^{\varepsilon}$ or a minimal annulus $\Sigma \in M$ with $\partial \Sigma=\alpha^{-\varepsilon} \cup \alpha^{\varepsilon}$.

Since $P^{0} \cap \partial T^{\varepsilon}$ is a regular value of $\Pi$ and the unique element in $\Pi^{-1}\left(P^{0} \cap \partial T^{\varepsilon}\right)$ is $P^{0} \cap T^{\varepsilon}$, it follows that $P^{0} \cap T^{\varepsilon}$ is on the boundary of one and only one of the compact arcs in $\Pi^{-1}(\Gamma)$. If $\Pi^{-1}\left(\alpha^{-\varepsilon} \cup \alpha^{\varepsilon}\right)$ contains more than one annulus in $M$, there must be at least one arc in $\Pi^{-1}(\Gamma)$ whose endpoints are distinct minimal annuli, $\Sigma, \Sigma^{\prime}$ in $\Pi^{-1}\left(\alpha^{-\varepsilon} \cup \alpha^{\varepsilon}\right) \subset M$. We now consider the index of the stability operator associated to the second variation of area. It is a function along the compact arc joining $\Sigma$ to $\Sigma^{\prime}$. As pointed out by White (see [27, Theorem 5.3]), the indices of $\Sigma$ and $\Sigma^{\prime}$ must have opposite parity (one is even, the other odd). In particular, one of the annuli $\Sigma, \Sigma^{\prime}$ has odd index and hence is unstable. But we have shown above that these annuli must be stable. Hence, $\Pi^{-1}\left(\alpha^{-\varepsilon} \cup \alpha^{\varepsilon}\right)$ contains a single annulus. This completes the proof of uniqueness.

Remark 2. In fact, the minimal annulus produced in Lemma 2 is the unique minimal surface in $T^{\varepsilon}$ with boundary $\alpha^{\varepsilon} \cup \alpha^{-\varepsilon}$. A simple consequence of a geometric version of Dehn's lemma [21] is the following. Suppose $M$ is a Riemannian three-manifold that is homeomorphic to a solid torus. Assume $M$ has nonnegative mean curvature. If $\gamma_{1}$ and $\gamma_{2}$ are two pairwise-disjoint simple closed curves in $\partial M$ that are homotopically nontrivial in $M$, and $\gamma_{1} \cup \gamma_{2}$ bounds a unique, embedded minimal annulus $A$ in $M$, then $A$ is the unique compact, branched, minimal surface in $M$ with this boundary. This result shows that the uniqueness statement of Lemma 3 holds even if one considers compact, branched minimal surfaces.

We are now in a position to describe a technical result that will be used in the following section. Let $\gamma_{t}$ be the circle of radius $t$ in $P^{0}$, centered at the origin. Let $C_{t}^{\varepsilon}$ be the stable catenoid whose boundary circles consist of the vertical translates of $\gamma_{t}$ by $(0,0, \pm \varepsilon)$. Let $\Delta_{\varepsilon}$ be the solid torus bounded by subsets of $C_{2}^{\varepsilon}, C_{4}^{\varepsilon}, P^{\varepsilon}$, and $P^{-\varepsilon}$. Note that $\partial \Delta_{\varepsilon}$ consists of two planar annuli and the two catenoids $C_{2}^{\varepsilon}, C_{4}^{\varepsilon}$. Let $K^{0} \subset P^{0}$ be the annulus bounded by $\gamma_{2}$ and $\gamma_{4}$. Let $\delta$ be any smooth Jordan curve in $K^{0}$ that is homotopic to $\partial K^{0}$ in $K^{0}$.

Proposition 1. For $\varepsilon>0$ sufficiently small, $\Delta_{\varepsilon}$ can be foliated by compact minimal annuli $A_{t}, 2 \leq t \leq 4$, with the following properties:

(1) $A_{t}=C_{t}^{\varepsilon}$, for $t$ sufficiently close to 2 or to 4 ;

(2) Each $A_{t}$ meets $P^{0}$ orthogonally;

(3) $A_{3}$ meets $P^{0}$ in a smooth Jordan curve that converges to $\delta$, in the $C^{0}$-norm, as $\varepsilon \rightarrow 0$.

Proof. Extend $\delta$ to a smooth foliation $\left\{\alpha_{t} \mid 2 \leq t \leq 4\right\}$ of $K^{0}$, with $\alpha_{t}=\gamma_{t}$, for $t$ close to $2, \alpha_{3}=\delta$, and $\alpha_{t}=\gamma_{t}$ for $t$ close to 4 . Let $\left\{\alpha_{t}^{ \pm \varepsilon}\right\}$ denote the corresponding foliation of $P^{ \pm \varepsilon} \cap \Delta^{\varepsilon}$. According to Lemma 2, for each fixed $t$, there exists an $\varepsilon_{2}>0$ such that for $\varepsilon<\varepsilon_{2}, \alpha_{t}^{-\varepsilon}$ bounds a stable minimal 


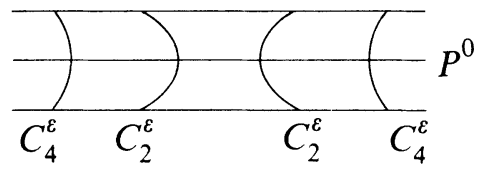

FIGURE 1

annulus, $\tilde{A}_{t}$, in the $\varepsilon$-neighborhood, $T_{t}^{\varepsilon}$ of $\alpha_{t}$. For $t$ near 2 or near 4 , it is clear that $\widetilde{A}_{t}=C_{t}^{\varepsilon}$. Let $\varepsilon_{3}$ be the infimum of the values of $\varepsilon_{t}, 2 \leq t \leq 4$. By Lemma $2, \varepsilon_{t}$ depends only upon $r_{\alpha_{t}}$, and $L_{\alpha_{t}}$. Hence, $\varepsilon_{3}>0$.

Suppose now that $\varepsilon<\varepsilon_{3}$. Since the catenoid $A_{2}=C_{2}^{\varepsilon}$ is stable, a small change of $\partial A_{2}$ to $\alpha_{t}^{ \pm \varepsilon}$ yields a nearby stable minimal annulus $A_{t}$. Since $A_{2}$ is stable and the variational vector field for $\left\{A_{t} \mid 2 \leq t\right.$ and close to 2$\}$ is never zero along $\partial A_{t}$, the variational vector field is never zero. Hence, $A_{t}$ moves away from itself as $t$ varies near 2 . It follows that the variation produces a foliation for $t$ near 2 .

Suppose $A_{s}$ is the limit of such a smooth variation of $A_{2}$ where each $A_{t}$ is contained in $T_{t}^{\varepsilon}$ of $\alpha_{t}$ for $t \geq 2$. Then not only is $A_{s} \subset T_{s}^{\varepsilon}$ but Theorem 2 in [21] guarantees that $A_{s}$ is transverse to $T_{3}^{\varepsilon}$. By Lemma 2, $A_{s}$ is stable and we can repeat the above argument to extend the previously defined foliation past $A_{s}$. In this way, we produce a smooth foliation $A_{t}, 2 \leq t \leq 4$. Since $A_{4}$ is a stable catenoid, $A_{4}=C_{4}^{\varepsilon} \subset \partial \Delta_{\varepsilon}$. Since all the leaves $A_{t}$ are contained in $\Delta_{\varepsilon}$ (a simple application of the maximum principle), these annuli form a smooth foliation of $\Delta_{\varepsilon}$.

Let $\hat{A}_{t}$ denote the image of $A_{t}$ under reflection through $P^{0}$. Since $\Delta_{\varepsilon}$ is invariant under reflection through $P^{0}, \widehat{A}_{t} \subset \Delta_{\varepsilon}$. If $\widehat{A}_{t} \neq A_{t}$, then there exists a largest or smallest $t_{0} \neq t$ such that $\widehat{A}_{t} \cap A_{t_{0}} \neq \varnothing$ which is impossible by the maximum principle. This shows $\widehat{A}_{t}=A_{t}$, and hence meets $P^{0}$ orthogonally. Let $\alpha_{2}^{0}=A_{2} \cap P^{0}$. By making $\varepsilon_{3}$ smaller, if necessary, we can insure that $\alpha_{2}^{0}$ is $C^{0}$-close to $\delta=\alpha_{2}$. This completes the proof the proposition.

Remark 3. By choosing $\delta$ to be a curve in $K^{0}$ that intersects the positive $x_{1}$ axis twice, transversally, and the negative $x_{1}$-axis only once, we can insure the existence of a point $p_{0} \in \delta$ where the tangent line to $\delta$ passes through the origin. At a point, $p_{1}$, of $\delta$ at maximum distance from the origin, the line segment $\overline{0 p_{1}}$ meets the tangent line to $\delta$ orthogonally. Thus, on a subarc of $\delta$ from $p_{0}$ to $p_{1}$, the cosine of the angle between the tangent line to $\delta$ and the position vector of $\delta$ must take on either all values between 0 and 1 or all the values between -1 and 0 . If $A_{t}, 2 \leq t \leq 4$, is the foliation produced in Proposition 1 , and $\varepsilon>0$ is small, $\alpha_{3}^{0}=A_{3} \cap P^{0}$ must have these same properties. Noting that the tangent line to $\alpha_{3}^{0}$ at $p$ is the intersection of $P^{0}$ with the tangent space to $A_{3}$, we may use these observations and Proposition 1 to conclude the following. 

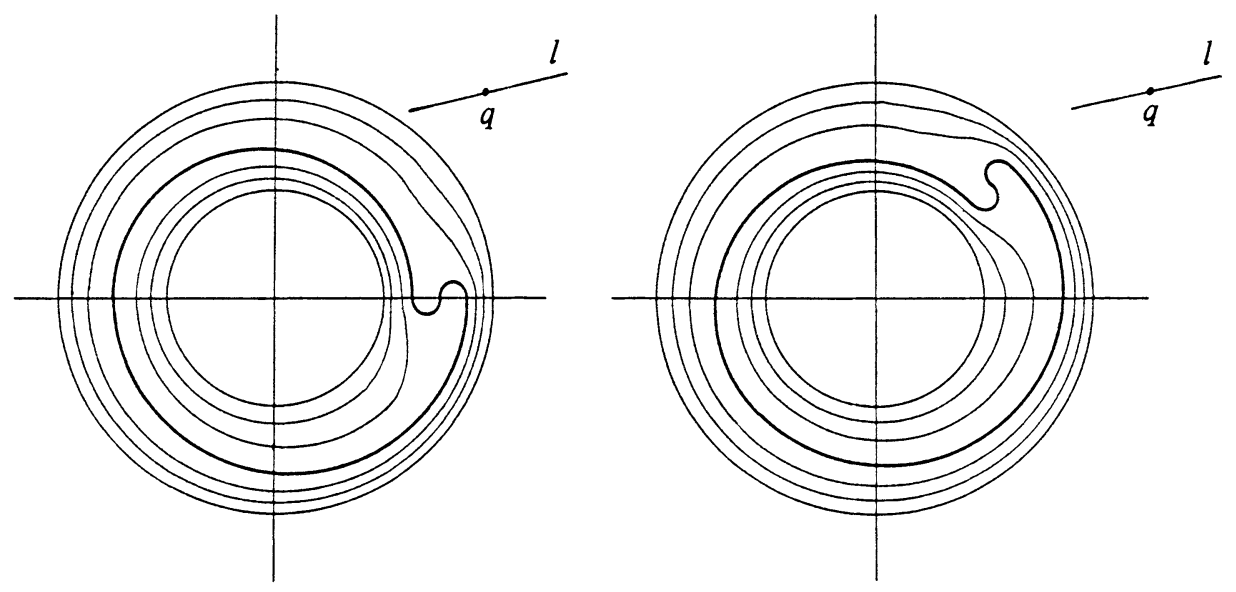

FIGURE 2

Corollary 1. For $\varepsilon>0$ sufficiently small, there exists a foliation $\left\{A_{t}\right\}$ of $\Delta_{\varepsilon}$ whose leaves satisfy all the conditions of Proposition 1 as well as

(4) for any $q \in P^{0}-\{0\}$, and any line l through $q,|q|>4$, we may rotate $\Delta_{\varepsilon}$ around the $x_{3}$-axis so that $\overline{0 q}$ intersects $\alpha_{3}^{0}=A_{3} \cap P^{0}$ in a point, $p$, where $T_{p} A_{3} \cap P^{0}$ is a line parallel to $l$.

See Figure 2.

\section{The Cone Lemma}

Let $X_{c}$ be the cone in $\mathbf{R}^{3}$ defined by the equation

$$
x_{1}^{2}+x_{2}^{2}=\left(x_{3} / c\right)^{2}, \quad c \neq 0 .
$$

The complement of $X_{c}$ consists of three components, two of which are convex. We label the third region $W_{c}$ and note that $W_{c}$ contains $P^{0}-\{0\}$, where $P^{0}=\left\{x_{3}=0\right\}$. See Figure 3. Suppose $M$ is a noncompact, properly immersed minimal surface, with compact boundary, that is disjoint from $X_{c}$. According to Theorem 3 of [10], the convex hull of $M$ is either a plane, a slab, a halfspace, or all of $\mathbf{R}^{3}$. Therefore, $M$ cannot lie in either of the convex components of $\mathbf{R}^{3}-X_{c}$. From this it follows that for such surfaces, the assumptions " $M \subset W_{c}$ " and " $M \cap X_{c}=\varnothing$ " are equivalent.

Note that as $c \rightarrow 0, X_{c}-\{0\}$ collapses to a double covering of $P^{0}-\{0\}$. Note also that any horizontal plane or vertical catenoid is eventually disjoint from any $X_{c}$, no matter how small $c$ is. (By "eventually" we mean "outside of a compact set".) Since any minimally embedded annular end of finite total curvature is asymptotic to a plane or a catenoid, it follows that, after suitable rotation, such an end is eventually disjoint from any $X_{c}$. It is easy to see that a minimally immersed end of finite total curvature with a horizontal limit tangent 


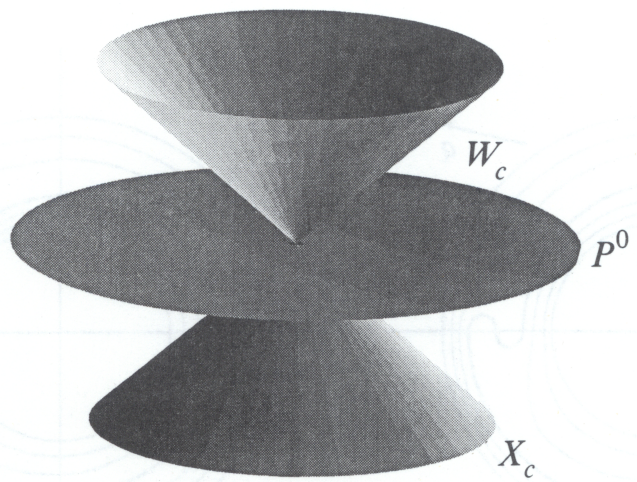

FIGURE 3

plane is also eventually disjoint from every $X_{c}$. (See [14].) The main result of this section shows that this property comes close to characterizing annular ends with finite total curvature.

Lemma 4 (The Cone Lemma). If a properly immersed minimal surface with compact boundary is eventually disjoint from a sufficiently shallow cone, then each annular end of the minimal surface has finite total curvature.

Proof. In order to prove the lemma, it is sufficient to establish the following statement for some cone $X_{c}, c>0$. If $M$ is an annular end of a properly immersed minimal surface, and $M$ is disjoint from $X_{c}$, then $M$ has finite total curvature. We will exhibit such a $c>0$.

We begin by normalizing the problem. From the discussion preceding the statement of the lemma, we know that $M \cap X_{c}=\varnothing$ if and only if $M \subset W_{c}$.

Let $C(1)$ be vertical catenoid with waist-circle of radius 1 and denote by $C$ the compact component of $W_{c} \cap C(1)$. Choose $c>0$ small enough so that $C$ is a radial graph and foliate $W_{c}$ by the leaves $\{t \cdot C\}, 0<t<\infty$. For convenience, we write $C_{t}$ for $t \cdot C$.

Claim 1. After a homothetic shrinking $M$ (but not of the foliation) and a discarding of a compact subset of $M$ :

(i) $\partial M \subset C_{1}=C$;

(ii) $M \subset \bigcup_{1 \leq t<\infty} C_{t}$;

(iii) $M \cap C_{t}$ consists of a single closed immersed curve for $t \geq 1$.

Proof of Claim 1. Choose $T_{0}$ large enough so that $\partial M$ lies in the bounded component of $W_{c}-C_{T_{0}}$. Without loss of generality, we may assume that $C_{T_{0}}$ intersects $M$ transversally. Denote by $Z$ the closure of the unbounded component of $W_{c}-C_{T_{0}}$; that is, $Z=\bigcup_{t \geq T_{0}} C_{t}$. Define $f: Z \rightarrow\left[T_{0}, \infty\right)$ to be the function whose level set at $t$ is $C_{t}$. Since the $C_{t}$ are minimal surfaces, the maximum principle implies that $\left.f\right|_{M \cap Z}$ has no interior maxima or minima. Moreover, the intersection of two minimal surfaces in a neighborhood of a point of tangency consists of $j$ curves, $j \geq 2$, intersecting at that point in equal angles. This implies that $\left.f\right|_{M \cap Z}$ has only index -1 critical points with 


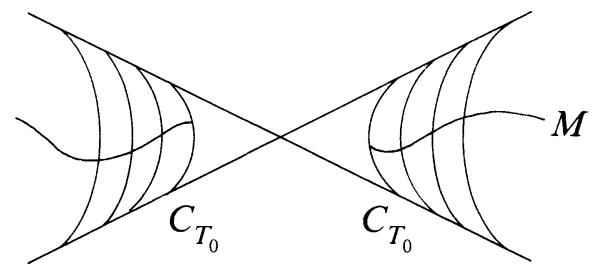

FIGURE 4

multiplicity equal to $j-1$. (See [20, Lemma 2] and also [15].) Therefore, $f$ may have at most $k-1$ critical points, where $k$ is the first Betti number of $M \cap Z$, by elementary Morse theory. Consequently, outside of a compact subset of $M \cap Z,\left.f\right|_{M \cap Z}$ is free of critical points. This means that there exists a $T_{1}>0$ such that for $t \geq T_{1}, C_{t} \cap M$ consists of a finite number of closed immersed curves. Since $M$ has one end, each $C_{t} \cap M, t \geq T_{1}$, must consist of a single closed immersed curve.

By discarding the compact subsurface $M \cap\left(\bigcup_{t \leq T_{1}} C_{t}\right)$ and rescaling by a factor of $T_{1}^{-1}$, we satisfy conditions (i), (ii), and (iii).

Because $M$ is properly immersed and projection from $W_{c}$ to $P^{0}-\{0\}$ is also proper, the projection $\Pi: M \rightarrow P^{0}-\{0\}$ is a proper map.

Claim 2. The mapping $\Pi$ is a submersion outside of a compact set, provided $c>0$ is sufficiently small.

Before proving Claim 2, we will show that the lemma follows from it. By a result of Osserman [22, Theorem 9.5] the normals to $M$ either take on all values, except for at most a set of capacity zero, or they possess unique limiting value. In the latter case, $F$ must have finite total curvature. But Claim 2 implies that, outside of some compact set, the normals to $M$ take on values in a hemisphere, so the first case of Osserman's result is precluded. Hence, $M$ has finite total curvature.

Proof of Claim 2. In this proof, we will need, at several points, to restrict the size of $c>0$. At each point, we will continue to assume that $M \subset W_{c}$. Let $\Delta:=\Delta_{\varepsilon}$ be the foliated annulus produced in Proposition 1. Reduce the size of $c$ so that the $\Delta$ has its top and bottom boundaries disjoint from $W_{c}$. Let $K$ be the intersection of $W_{c}$ with the vertical cylinder over the disk of radius 4 in $P^{0}$. Note $\Delta \cap W_{c} \subset K$. Shrink $c>0$ even more, if necessary, so that the following is true. If the distance from $q \in K$ to $P^{0}$ is $\tau$, then the vertical translation of $\Delta$ by $\tau$ has the property that its top and bottom boundaries are disjoint from $W_{c}$.

Suppose now that $\Pi: M \rightarrow P^{0}$ is not a submersion outside of some compact set. This is equivalent to the statement that the points on $M$ with vertical tangent plane form an unbounded set. In particular, there is a point $p \in M-K$, whose tangent plane is vertical.

According to Corollary 1 , we may rotate $M$ about the vertical axis so that the following holds. If $\hat{p}$ is the projection of $p$ onto $P^{0}$, the line $\overline{0 \hat{p}}$ intersects $\alpha_{2}$ 


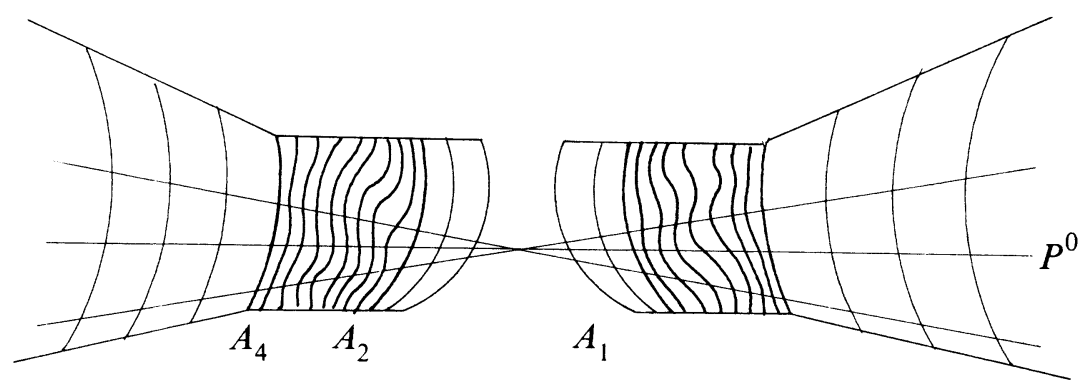

FIGURE 5

at a point where the tangent line to $\alpha_{2}$ is parallel to $T_{p} M \cap P^{0}$. We perform this rotation of $M$ and shrink $M$ so that $\hat{p}$ actually lies on $\alpha_{2}$. Since the original $M$ satisfied the conditions of Claim 1, and the foliation $\left\{C_{t} \mid 0<t<\infty\right\}$ is rotationally symmetric, it follows easily that the modified $M$ also satisfies condition (iii) of Claim 1. We also discard $M \cap \bigcup_{t<1} C_{t}$. We will refer to this modified surface as $M$. It is clear that to prove the claim, it is sufficient to prove it for this modified surface.

Vertically translate $\Delta$ so that $\hat{p}$ coincides with $p$, and label this translated torus $\widehat{\Delta}$. Also translate the foliation $A_{t}$ of $\Delta$ to be a foliation in $\widehat{A}_{t}$ of $\widehat{\Delta}$. Recall that we have chosen $c>0$ small enough so that the top and bottom boundaries of $\widehat{\Delta}$ are disjoint from $W_{c}$. Also recall that for $t$ near 2 and 4 , the leaves of the foliation of $\widehat{\Delta}$ are catenoids. Make $c$ smaller, if necessary, to insure that these catenoids are radial graphs.

We will now extend $\widehat{A}_{t}$ to be a smooth foliation of a region that contains $\bigcup_{4 \leq t \leq \infty} C_{t}$. Let $\widehat{A}_{t}=\frac{t}{4} \widehat{A}_{4}, t \geq 4$, be the homothetic expansion of $\widehat{A}_{4}$. (See Figure 5.) The boundary $\partial \widehat{A_{2}}$ consists of two concentric circles. By making $c>0$ smaller if necessary, we may insure that $C$ is a subset of a stable catenoid, $\widehat{C}$, whose boundaries are concentric circles exterior to $W_{c}$ on the same planes that contain $\partial \widehat{A}_{2}$. We interpolate between $\partial \widehat{A}_{2}$ and $\partial \widehat{C}$ with a smooth family, each member of which is a pair of circles centered on the vertical axis. The distance between circles in each pair is an increasing function of $t, 1 \leq t \leq 2$. Note that each pair of circles bounds a unique stable catenoid. Label that catenoid $\widehat{A}_{t}, 1 \leq t \leq 2$. It is evident that this family may be chosen to insure that the resulting foliation $\left\{\widehat{A}_{t} \mid 0 \leq t<\infty\right\}$ is smooth. By construction, $M \subset \bigcup_{1 \leq t<\infty} \widehat{A}_{t}, \partial M \subset \widehat{A}_{1}$, and $\widehat{A}_{3}$ is tangent to $M$ at $p$.

Let $h$ be the smooth function, defined on the union of the leaves $\widehat{A}_{t}$, whose level set at $t$ is $\hat{A}_{t}$. Restriction of $h$ to $M$ yields a proper function $M$ that satisfies $\left.h\right|_{M} \geq 1$ and is equal to 1 precisely on $\partial M$. Repeating the argument in the proof of Claim 1 will show that all the critical points of $h$ have index +1 , possibly with multiplicity. However, $M$ is an annulus, so by elementary Morse theory, it follows that $\left.h\right|_{M}$ can have no critical points. But $\widehat{A}_{3}$ is tangent to $M$ 
at $p \in M$, which shows that $p$ is a critical point of $\left.h\right|_{M}$. This contradiction completes the proof of Claim 2 and also of the lemma.

\section{STANDARD BARRIERS AND THE PROOF OF THE ANNULAR END THEOREM}

For convenience, in the proofs and discussions of this section, we introduce the notion of a standard barrier.

Definition 1. A standard barrier in $\mathbf{R}^{3}$ is one of the following two minimal surfaces with boundary: the complement of a disk in a plane in $\mathbf{R}^{3} ;$ a component of the complement of a simple, closed, homotopically nontrivial curve on a catenoid.

We will say that a surface $M \subset \mathbf{R}^{3}$ admits a standard barrier if it is disjoint from some standard barrier. As in the previous section, we will use the word "eventually" to mean "outside of some sufficiently large compact set of $\mathbf{R}^{3}$." Thus, two surfaces $M \subset \mathbf{R}^{3}$ and $N \subset \mathbf{R}^{3}$ are "eventually disjoint" if they have compact intersection. It is straightforward to see that $M$ admits a standard barrier if and only if it is eventually disjoint from some standard barrier.

Given a standard $S$ and a ball $B$ large enough to contain $\partial S$, it is clear that $S-B$ divides $\mathbf{R}^{3}-B$ into two components. Two surfaces $M, N \subset \mathbf{R}^{3}$ will be said to be separated by a standard barrier if such an $S$ and $B$ can be found so that $M$ and $N$ eventually lie in different components of $\mathbf{R}^{3}-(B \cup S)$.

Two disjoint standard barriers divide the complement of a sufficiently large ball $B \subset \mathbf{R}^{3}$ into three components, only one of which contains portions of both barriers on its boundary. A surface $M \subset \mathbf{R}^{3}$ that eventually lies in such a component will be said to lie between two standard barriers. After a rotation of $\mathbf{R}^{3}$, if necessary, the region of $\mathbf{R}^{3}$ between two standard barriers eventually lies in the complement of any cone $X_{c}=\left\{x_{1}^{2}+x_{2}^{2}=\left(x_{3} / c\right)^{2}\right\}$ (in the component that contains $P^{0}-\{0\}$ ) for any $c>0$, no matter how small. It follows from the Cone Lemma (Lemma 4 of $\S 3$ ) that

Corollary 2. If a properly immersed minimal annulus in $\mathbf{R}^{3}$ with compact boundary lies between two standard barriers, it must have finite total curvature.

Our strategy in proving the Annular End Theorem (Theorem 1 below) is to trap ends between standard barriers. The next lemma contains the critical technical construction.

Lemma 5. Suppose $M$ is a properly embedded, piecewise-smooth surface that is a smooth minimal surface outside of some ball and that has at least two ends. Let $\gamma: \mathbf{R} \rightarrow M$ be a proper curve that diverges into two distinct ends of $M$, depending on whether $t \rightarrow+\infty$ or $t \rightarrow-\infty$. Then $M$ admits a standard barrier whose boundary has linking number 1 with $\gamma$.

Proof. Let $B \subset \mathbf{R}^{3}$ be a ball large enough to contain the nonsmooth, nonminimal portions of $M$, and expand it, if necessary, so that the ends of $M$ in question correspond to distinct components of $M-B$. If one has such a ball, any larger one will have the same property. We may also choose $B$ so that $\partial B$ intersects $M$ transversally. Suppose that $M_{1}$ and $M_{2}$ are the two components 


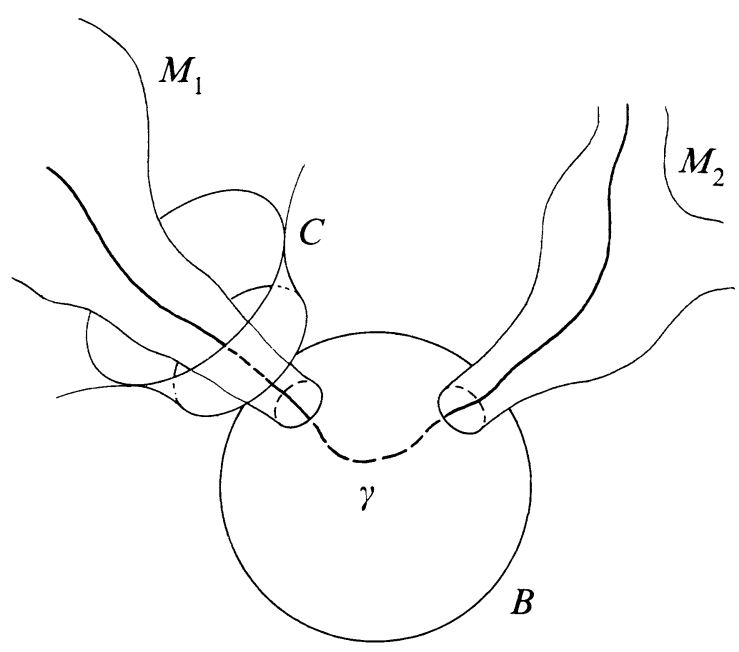

FIGURE 6

of $M-B$ that contain the unbounded components of $\gamma-B$. Since the proper arc $\gamma$ intersects $\partial M_{1}$ an odd number of times, we can choose exactly one component of $\mathbf{R}^{3}-M$ whose closure, $\mathscr{N}$, has the following property. The arc $\gamma$ has odd linking number with any 1 -cycle in $\operatorname{Int}(\mathscr{N})$ homologous to $\partial M_{1}$ in $\mathscr{N}$. Note that $\partial M_{1}$ is not homologous to zero in $\mathscr{N}$.

Let $\Sigma_{1} \subset \cdots \subset \Sigma_{n} \subset \cdots$ be an exhaustion of $M_{1}$ by smooth compact subdomains, with $\partial M_{1} \subset \partial \Sigma_{1}$. Let $\widetilde{\Sigma}_{i}$ denote a least-area integral current in $\mathscr{N}$ with boundary $\partial \Sigma_{i}$, which is $Z_{2}$-homologous to $\Sigma_{i}$. Since $\Sigma_{i} \cup \widetilde{\Sigma}_{i}$ is a boundary in $\mathscr{N}, \widetilde{\Sigma}_{i}$ is orientable. Interior regularity of least-area currents (see, for example, [25]) shows that $\widetilde{\Sigma}_{1} \cap \operatorname{Int}(\mathscr{N})$ is a regular embedded minimal surface. Since $\partial \mathscr{N}-\partial B$ has zero mean curvature, the maximum principle and the unique continuation property for minimal surfaces imply that either $\widetilde{\Sigma}_{i} \cap(\mathcal{N}-\partial B)$ is regular and equals $\partial \Sigma_{i}$ or $\widetilde{\Sigma}_{i} \subset M_{1}$. Standard compactness theorems imply that a subsequence of the surfaces $\left\{\widetilde{\Sigma}_{i}\right\}$ converge to a leastarea orientable surface $\Sigma \subset \mathscr{N}$ with $\partial \Sigma=\partial M_{1}$. Suppose, for the moment, $\Sigma \cap M=\partial \Sigma$.

The surface $\Sigma-\partial B$ is a stable, properly embedded, orientable minimal surface in $\mathbf{R}^{3}$ with compact boundary and hence has finite total curvature (see, [19, Theorem 1] as well as [7j). Hence, $\Sigma$ has a finite number, say $n$, of ends, and each end is asymptotic to a plane or to a catenoid. Let $S_{R}$ be the sphere of radius $R$ centered at the origin. For $R$ sufficiently large, $\Sigma \cap S_{R}$ consists of $n$ parallel almost-great-circles, each of which is the boundary of one of the annular ends of $\Sigma$ (see [14]).

By our choice of $\mathcal{N}, \gamma$ has odd linking number with one of the curves in $\Sigma \cap S_{R}$, and hence has linking number 1 with one of the annular ends of $\Sigma-B_{R}$. Call this annular end $F$.

In [17], a strong version of the maximum principle is proved, i.e., if two properly immersed minimal surfaces with compact boundaries are disjoint, they 
stay a bounded distance apart. Therefore, $F$ stays a positive distance from $M .^{3}$ On the other hand, $F$ is asymptotic to an end, $C^{\prime}$, of a plane or a catenoid. Hence, $C^{\prime}$ contains a subend, $C$, whose boundary is a circle that has linking number 1 with $\gamma$. Moreover, $C$ is contained in the interior of $\mathscr{N}$. This proves the lemma in the case $\Sigma \cap M=\partial \Sigma$.

In case $\partial \Sigma \varsubsetneqq \Sigma \cap M$, the maximum principle implies that $\Sigma=M_{1}$, which means that $\gamma \cap M_{1}$ is eventually contained in a catenoid-type end or a plane-type end, say $C^{\prime \prime} \subset \Sigma$. This is, in fact, the easier case and can be treated directly, but we prefer to reduce it to the previous case. We may choose $C^{\prime \prime}$ so that it is as close as desired to a standard barrier. Moving $C^{\prime \prime}$ a small amount in the direction of its limiting normal produces a minimal surface that is disjoint from $M_{1}$ and its boundary has linking number with $\gamma$ equal to either 0 or 1 . Move in the direction that makes the linking number equal to 1 . The strong maximum principle shows that if $C^{\prime \prime}$ is moved a small amount, then it is also disjoint from $M$. We can now apply the argument in the previous case to complete the proof.

Corollary 3. Suppose $M_{1}, M_{2}$, and $M_{3}$ are three pairwise-disjoint, properly embedded minimal surfaces in $\mathbf{R}^{3}$, each of which has compact boundary and one end. Then at least one of the surfaces lies between two standard barriers.

Proof. Choose a ball $B \subset \mathbf{R}^{3}$ that is big enough to contain $\bigcup_{i} \partial M_{i}$. The ball can be chosen to intersect $\bigcup_{i} M_{i}$ transversally. After removal of $B \cap M_{i}$ from each $M_{i}$, we may assume that $\partial M_{i} \subset \partial B$. The curves $\bigcup_{i} \partial M_{i}$ bound a region $S$ on $\partial B$ with the property that the boundary of at least one component of $S$ touches the boundary of more than one of the $M_{i}$. We will refer to this component as $\mathscr{S}$ and relabel the $M_{i}$, if necessary, so that both $\partial \mathscr{S} \cap \partial M_{1}$ and $\partial \mathscr{S} \cap \partial M_{2}$ are nonempty.

Let $M=S \bigcup_{i} M_{i}$. We intend to apply Lemma 5 to $M$. Toward that end, choose a proper curve $\gamma: \mathbf{R} \rightarrow M$, with $\gamma(\mathbf{R}) \cap S$ consisting of a single connected arc in $\mathscr{S}$ from $\partial M_{1}$ to $\partial M_{2}$. We may assume that $\gamma$ diverges in $M_{1}$ (resp. $M_{2}$ ) as $t \rightarrow+\infty$ (resp. $\left.t \rightarrow-\infty\right)$ ). By Lemma 5, there exists a standard barrier disjoint from $M$ whose boundary has linking number 1 with $\gamma$.

We now expand the ball $B$ to be large enough to contain the boundary of this barrier and discard from each $M_{i}$ the subset $M_{i} \cap B$. Similarly, let $C_{1}$ be the component of the barrier exterior to $B . C_{1}$ is still a barrier for $M$, and $\gamma$ has linking number 1 with $\partial C_{1}$. Moreover, $C_{1}$ divides $\mathbf{R}^{3}-B$ into two components. Clearly, $M_{1}$ and $M_{2}$ are in different components. Without loss of generality, we may assume that $M_{3}$ is in the same components as $M_{1}$.

The curve $\partial C_{1}$ divides $\partial B$ into two disks. Let $D$ be the disk containing $\partial M_{1} \cup \partial M_{3}$. We now repeat the construction in the previous paragraph. This time, let $S^{\prime}$ be a region of $D$ bounded by $\partial C_{1} \cup \partial M_{1} \cup \partial M_{3}$. Elementary arguments show that $S^{\prime}$ has a component, say $\mathscr{S}^{\prime}$, with boundary points on both $\partial M_{1}$ and $\partial M_{3}$. Let $M^{\prime}=M_{1} \cup M_{3} \cup C_{1} \cup S^{\prime}$. Choose a proper arc

\footnotetext{
${ }^{3}$ The use of this version of the maximum principle could be avoided by allowing barriers to be embedded minimal ends that are asymptotic to catenoids or planes.
} 


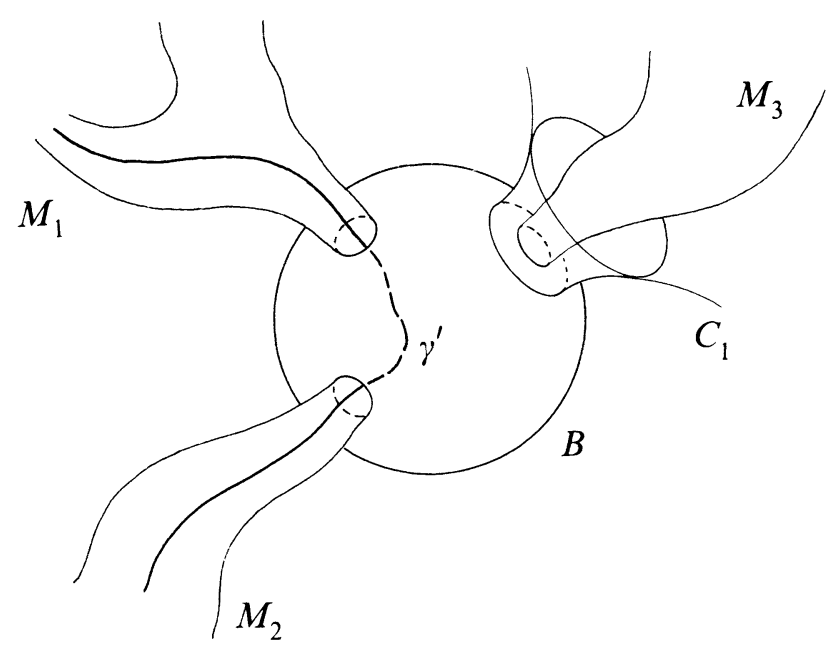

FIGURE 7

$\gamma^{\prime} \subset M^{\prime}$ whose intersection with $S^{\prime}$ lies in $\mathscr{S}^{\prime}$ and consists of a connected arc from $\partial M_{1}$ to $\partial M_{3}$. (Note that, since $(\partial B-D) \cap \gamma^{\prime}=\varnothing$ and $\partial D=\partial C_{1}, \gamma^{\prime}$ has linking number 0 with $\partial C_{1}$.) Lemma 5 implies that there exists another standard barrier, $C_{2}$, that is disjoint from $M_{1} \cup M_{3} \cup C_{1} \cup S$ and whose boundary has linking number 1 with $\gamma^{\prime}$.

Expand $B$ again so that $\partial C_{2} \subset B$. It is possible to do this so that $\partial B$ meets $M$ transversally. Note that $C_{2} \cap \partial B$ is a single closed curve. Again, we discard from $M_{1}, M_{2}, M_{3}, C_{1}$, and $C_{2}$ the intersection of those surfaces with $B$. Therefore, all of these surfaces have their boundaries on $\partial B$.

The barrier $C_{2}$ divides $\mathbf{R}^{3}-B$ into two region, as does the barrier $C_{1}$. Since they are disjoint, $C_{1} \cup C_{2}$ divides $\mathbf{R}^{3}-B$ into three components. Let $T_{1}$ (resp. $\left.T_{2}\right)$ be the component of $\left(\mathbf{R}^{3}-B\right)-\left(C_{1} \cup C_{2}\right)$ whose boundary contains $C_{1}$ but is disjoint from $C_{2}$ (resp. contains $C_{2}$ but is disjoint for $C_{1}$ ). Let $F$ be the third component, whose boundary contains $C_{1} \cup C_{2}$. Since $\gamma^{\prime}$ has linking number 1 with $\partial C_{2}, C_{2}$ must separate $M_{1}$ from $M_{3}$. But clearly, $M_{1} \cup M_{3} \subset T_{1} \cup F$. Hence, either $M_{1}$ or $M_{3}$ lies in $F$. That is, either $M_{1}$ or $M_{3}$ lies between two standard barriers.

Remark 4. Lemma 5 and Corollary 3 hold even when the minimal surfaces in question are properly immersed rather than properly embedded. The proofs are essentially the same as the proof of the embedded case. See [17] for these types of arguments.

Theorem 1 (The Annular End Theorem). If $M$ is a properly embedded minimal surface in $\mathbf{R}^{3}$, then at most two distinct annular ends of $M$ can have infinite total curvature.

Proof. If $M$ has two or fewer annular ends, there is nothing to prove. If $M$ has three or more annular ends, we apply Corollary 3 to any choice of three annular ends of $M$. It implies that one of them lies between two standard 
barriers. But by Corollary 2, this end must have finite total curvature. Thus, $M$ can have at most two annular ends of infinite total curvature.

Corollary 4. Suppose $M$ is a properly embedded minimal surface in $\mathbf{R}^{3}$. Then $M$ can have at most two annular ends that are not conformally diffeomorphic to a once-punctured disk. In particular, if $M$ has finite topology, then $M$ is conformally equivalent to a closed Riemann surface from which a finite number of points, and zero, one, or two pairwise-disjoint compact disks have been removed.

Proof. By the classification of conformal structures of annuli, each annular end of $M$ is conformally diffeomorphic to $\{z \in \mathbf{C}|R<| z \mid \leq 1\}$ for some $R>0$. Since an annular end of $M$ of finite total curvature is conformally diffeomorphic to $D-\{\overrightarrow{0}\}[22$, Theorem 9.3], $M$ can have at most two annular ends that are not conformally diffeomorphic to a punctured disk.

\section{REFERENCES}

1. M. Anderson, Curvature estimates for minimal surfaces, Ann. Sci. École Norm. Sup. 18 (1985), 89-105.

2. L. Barbosa and M. do Carmo, On the size of a stable minimal surface in $\mathbf{R}^{3}$, Amer. J. Math. 19 (1976), 515-528.

3. M. Callahan, D. Hoffman, and W. H. Meeks III, Embedded minimal surfaces with four ends, 1989, preprint.

4. __ The structure of singly-periodic minimal surfaces, Invent. Math. (to appear).

5. C. Costa, Example of a complete minimal immersion in $\mathbf{R}^{3}$ of genus one and three embedded ends, Bol. Soc. Brasil. Mat. 15 (1984), 47-54.

6. Y. Fang and W. Meeks, Some global properties of complete minimal surfaces in $\mathbf{R}^{3}$, Topology (to appear).

7. D. Fischer-Colbrie, On complete minimal surfaces with finite Morse index in 3-manifolds, Invent. Math. 82 (1985), 121-132.

8. D. Hoffman and W. H. Meeks III, One-parameter families of embedded minimal surfaces, 1989, preprint.

9. ___ Properly embedded minimal surfaces of finite topology, Ann. of Math. (2) (to appear).

10. __ The strong halfspace theorem for minimal surfaces, 1987, preprint.

11. A variational approach to the existence of complete embedded minimal surfaces, Duke $\mathrm{J}$. Math. 57 (1988), 877-893.

12. __ A complete embedded minimal surface with genus one, three ends and finite total curvature, J. Differential Geom. 21 (1985), 109-127.

13. _ Properties of properly embedded minimal surfaces of finite total curvature, Bull. Amer. Math. Soc. 17 (1987), 296-300.

14. L. Jorge and W. H. Meeks III, The topology of complete minimal surfaces of finite total Gaussian curvature, Topology 22 (1983), 203-221.

15. T. C. Kuo, On $C^{\infty}$-sufficiency of sets of potential functions, Topology 8 (1969), 167-171.

16. W. H. Meeks III and H. Rosenberg, The global theory of doubly-periodic minimal surfaces, Invent. Math. (to appear).

17. __ The strong maximum principle for complete minimal surfaces in flat 3-manifolds, 1988, preprint.

18. _ The geometry of periodic minimal surfaces, 1988, preprint.

19. W. H. Meeks III and S. T. Yau, The topological uniqueness theorem for minimal surfaces of finite type, 1987, preprint. 
20. __ The classical Plateau problem and the topology of three-dimensional manifolds, Topology 21 (1982), 409-442.

21. 179 (1982), 151-168.

22. R. Osserman, A survey of minimal surfaces, 2nd ed., Dover, New York, 1986.

23. J. Pitts and H. Rubenstein, Existence of minimal surfaces of bounded topological type in threemanifolds, Proc. Centre Math. Anal. Austral. Nat. Univ., Vol. 10, Canberra, Australia, 1987.

24. R. Schoen, Uniqueness, symmetry, and embeddedness of minimal surfaces, J. Differential Geom. 18 (1983), 791-809.

25. L. Simon, Lectures on geometric measure theory, Proc. Centre Math. Anal. Austral. Nat. Univ., Vol. 3, Canberra, Australia, 1983.

26. S. Smale, An infinite dimensional version of Sards theorem, Amer. J. Math. 87 (1965), 861-866.

27. B. White, New applications of mapping degree to minimal surface theory, J. Differential Geom. 29 (1989), 143-162.

Department of Mathematics, University of Massachusetts, Amherst, Massachusetts 01003 